\title{
Structural Health Monitoring on Turbine Engines Using Microwave Blade Tip Clearance Sensors
}

\author{
Mark Woike, Ali Abdul-Aziz, and Michelle Clem \\ National Aeronautics and Space Administration \\ Glenn Research Center, Cleveland, Ohio 44135
}

\begin{abstract}
The ability to monitor the structural health of the rotating components, especially in the hot sections of turbine engines, is of major interest to the aero community in improving engine safety and reliability. The use of instrumentation for these applications remains very challenging. It requires sensors and techniques that are highly accurate, are able to operate in a high temperature environment, and can detect minute changes and hidden flaws before catastrophic events occur. The National Aeronautics and Space Administration (NASA) has taken a lead role in the investigation of new sensor technologies and techniques for the in situ structural health monitoring of gas turbine engines. As part of this effort, microwave sensor technology has been investigated as a means of making high temperature non-contact blade tip clearance, blade tip timing, and blade vibration measurements for use in gas turbine engines. This paper presents a summary of key results and findings obtained from the evaluation of two different types of microwave sensors that have been investigated for possible use in structural health monitoring applications. The first is a microwave blade tip clearance sensor that has been evaluated on a large scale Axial Vane Fan, a subscale Turbofan, and more recently on sub-scale turbine engine like disks. The second is a novel microwave based blade vibration sensor that was also used in parallel with the microwave blade tip clearance sensors on the same experiments with the sub-scale turbine engine disks.
\end{abstract}

\section{INTRODUCTION}

The capability to monitor the structural health of the rotating components in a gas turbine engine is essential to the aero community for improving engine safety and reliability. The use of instrumentation for these applications is very challenging. It requires sensors and techniques that are able to operate in a very high temperature environment, are highly accurate, and can detect minute structural changes before catastrophic events occur. NASA's Aviation Safety Program (AVSP) is investigating new sensor technologies and techniques for the in-situ structural health monitoring of gas turbine engines. As part of this project, microwave sensor technology is being investigated as a means of making high temperature non-contact blade tip clearance, blade tip timing, and blade vibration measurements for use in structural health monitoring applications in gas turbine engines.

Microwave based sensor technology has the potential to be highly accurate, able to operate in a high temperature environment, and not be affected by contaminants that are present in engines. The use of microwave blade tip clearance and blade vibration sensors is an emerging concept. As a means of better understanding the issues associated with this type of sensor, several experiments have been conducted to evaluate sensor performance on aero engine type applications. This included the use of microwave blade tip clearance sensors on a large scale Axial Vane Fan and a subscale Turbofan. More recently, microwave blade tip clearance sensors have been evaluated for their suitability in making crack detection measurements on sub scale turbine engine like disks.

This latest experiment involved machining a notch to simulate a crack on a subscale turbine engine disk and monitoring the vibration response measured by the microwave tip clearance sensors as the disk was rotated at speeds up to $12000 \mathrm{rpm}$ on the NASA Glenn Research Center's High Precision Spin Rig. The purpose of this experiment is to evaluate the $24 \mathrm{GHz}$ 
microwave sensors capability to make accurate and stable blade tip clearance measurements on rotating components operated at speeds up to $12000 \mathrm{RM}$, which is much higher than the speeds where previous evaluations were conducted at. In addition the experiment was also to investigate if the microwave sensors were sensitive and accurate enough to detect the minute changes in the disks whirl amplitude and phase caused by the presence of the simulated crack as predicted by the theoretical models. These measurements were compared to those acquired with high frequency capacitive blade tip clearance sensors which have been the standard sensor used in previous crack detection experiments. In addition, a novel microwave vibration measurement system was also used to demonstrate its capability in making non-contact vibration measurements on rotating components.

This paper presents a summary of key results and findings obtained from the previous evaluation experiments using the microwave blade tip clearance sensors on the Axial Vane Fan and Turbofan, along with the results of using the microwave blade tip clearance sensors and new microwave vibration sensor on an experiment conducted on subscale turbine engine disks.

\section{MICROWAVE BLADE TIP CLEARANCE / BLADE TIP TIMING SENSORS}

The ability to monitor the structural health of the rotating components, especially in the hot sections of turbine engines, is of major interest to the aero community in improving engine safety and reliability (Ref. 1). In addition, the active control and minimization of the gap between the rotating turbine blades and the stationary case of gas turbine engines is being sought as a means of increasing engine efficiency, reducing fuel consumption, reducing emissions, and increasing engine service life (Ref. 2). To address this need, microwave sensor technology has been investigated as a means of making high temperature non-contact blade tip clearance and tip timing measurements for use in structural health monitoring and active clearance control applications in turbine engines. This technology is appealing due to its high accuracy and its potential to operate at extremely high temperatures that are present in turbine engines. It is intended to use blade tip clearance to monitor blade growth \& wear and blade tip timing to monitor blade vibration \& deflection.

\subsection{SENSOR BACKGROUND AND THEORY}

NASA has worked with Radatec (now Meggitt Inc.) through the Small Business Innovation Research Program (SBIR) program for the development of microwave sensor technology for high temperature non-contact blade tip clearance and blade tip timing measurements. The initial development of the technology was accomplished through a phase II SBIR contract awarded in 2002. Further development of the technology was accomplished in 2004 through 2005 as part of NASA's Ultra Efficient Engine Technology (UEET) Program. A prototype first generation $5.8 \mathrm{GHZ}$ system was delivered as part of a phase III SBIR commercialization contract in 2007 and a second generation $24 \mathrm{GHZ}$ system along with upgraded electronics were delivered as part of subsequent follow-on contracts in 2009 and 2010 respectively.

The microwave blade tip clearance sensor operates essentially as a field disturbance device. The tip clearance probe contains both a transmitting and receiving antenna. The sensor emits a continuous microwave signal and measures the signal that is reflected off a rotating blade. The sensor measures the changes in the microwave field due to the blade passing through the field. The motion of the blade phase modulates the reflected signal and this reflected signal is compared to an internal reference. Changes in amplitude and phase directly correspond to changes in the distance to the blade. The time interval of when the blade passes through the field is measured to provide blade tip timing. More detailed information on the sensor's theory of operation can be found in References 3 to 5. The microwave blade tip clearance probes are made of high temperature material and are designed to operate in temperatures up to $900{ }^{\circ} \mathrm{C}$. The first generation probes (Fig. 1a) operate at $5.8 \mathrm{GHz}$ and can measure clearance distances up to one-half the radiating wavelength which is $25 \mathrm{~mm}(\sim 1.0 \mathrm{in}$.). The second generation probes (Fig. 1b) operate at $24 \mathrm{GHz}$ and can measure clearance distances up to $6 \mathrm{~mm}(\sim 0.25$ in.). The 5.8 $\mathrm{GHz}$ sensor is targeted for use on large rotating machinery such as land based power turbines or in the fan sections of aero gas turbine engines. The $24 \mathrm{GHz}$ sensor is being targeted for use in smaller rotating machinery applications such as the turbine and compressor sections of aero engines. This technology has an ultimate goal of obtaining clearance measurement 
accuracies approaching $+/-25 \mu \mathrm{m}(+/-\sim 0.001$ in.). A frequency response of up to $5 \mathrm{MHz}$ is typical, with up to $25 \mathrm{MHz}$ being possible with this technology which lends itself for use in structural health monitoring applications for the measurement of blade deflection and vibration.

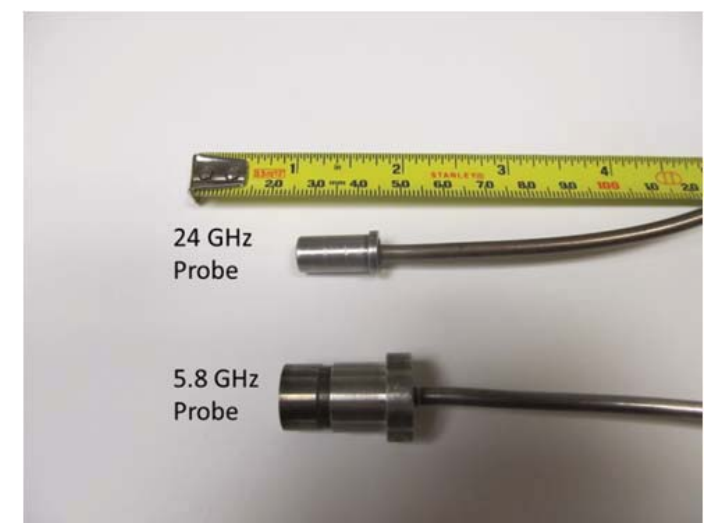

Figure 1. - Microwave blade tip clearance probes (Meggitt).

\subsection{EXPERIMENTAL RESULTS}

NASA's primary goal is to demonstrate this microwave blade tip clearance sensor technology on an actual gas turbine engine in a relevant high temperature environment. However, the use of microwave sensors for this application is an emerging concept. Techniques on their use and calibration needed to be understood and developed. In addition, the microwave sensor's accuracy and ability to make blade tip clearance and deflection measurements had to be assessed prior to use on actual engine hardware. As a means of better understanding the issues associated with the microwave sensors, a series of experiments were conducted to evaluate the sensor's performance on aero engine type applications. A summary of these experiments and their results are as follows.

The first generation $5.8 \mathrm{GHz}$ microwave sensors were used to a make blade tip clearance measurements on a large Axial Vane Fan and a subscale NASA Turbofan (Ref. 6). The purpose of the test on the large Axial Vane Fan (Fig. 2) was to develop the infrastructure required for the calibration of the sensors and the techniques for their use in the field to make clearance measurements on large rotating machinery. The motivation behind their use on the NASA Turbofan (Fig. 3) was to evaluate the first generation sensor's ability to acquire blade tip clearance data on an aero engine size test article and blades. Blade tip clearance data sets were acquired for several test runs of the NASA Turbofan. Data was acquired at a variable sampling rate that was synchronized to the fan's speed. Each measurement consisted of two revolutions of data with 10000 samples acquired per revolution. Figure 4 shows the individual blade clearances measured for several fan speeds. It is clearly noted from the polar plot that the tip clearances decreased as the fan speed is increased.

This result is expected and is due to the growth and expansion of the turbofan's composite blades as the fan operates at higher speeds. An average decrease of $0.22 \mathrm{~mm}$ ( $\sim 0.009$ in.) was observed as the rig speed was increased to $8875 \mathrm{rpm}$. The change in clearance detected in this experiment was within the range predicted for these blades. In addition, the change in tip clearances measured by the microwave sensors was nearly identical to previously recorded values obtained with capacitive clearance sensors. In previous test entries, changes in tip clearances of up to $0.22 \mathrm{~mm}(\sim 0.009$ in.) were also noted when the turbofan was operated over the same speed range. These two initial experiments served as test beds for the development of techniques and infrastructure required for the calibration of the sensors and successfully demonstrated the microwave clearance sensor's ability to make measurements on aero engine size hardware. 


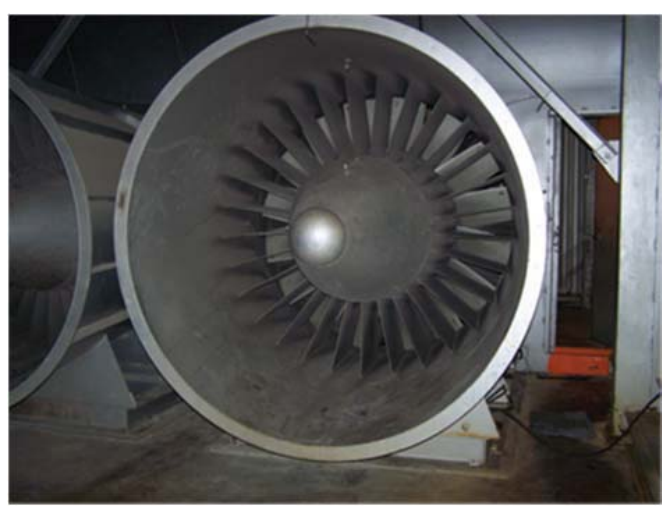

Figure 2. - Axial Vane Fan.

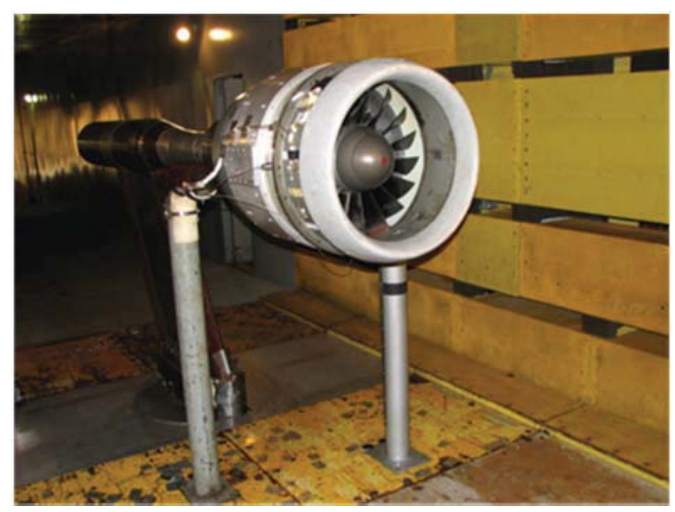

Figure 3. - NASA Turbofan.

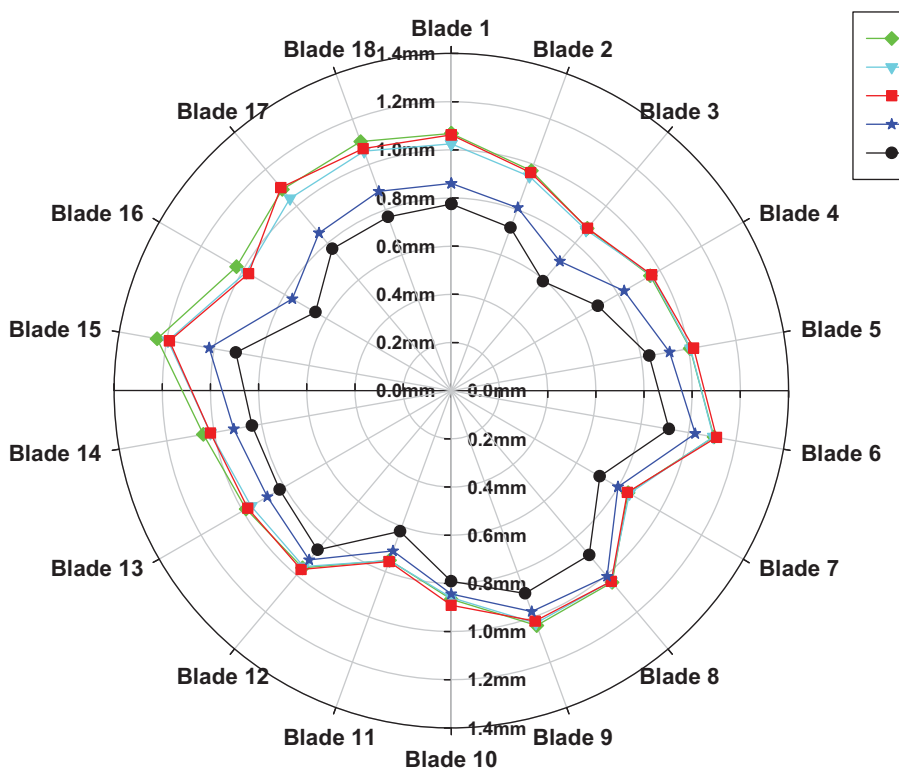

Figure 4. - Blade tip clearance polar plot for probe $\# 1,90^{\circ}$ location - NASA Turbofan experiment.

The second generation $24 \mathrm{GHz}$ sensors were previously used to make measurements on a 32 blade subscale turbine engine like disk and on an actual compressor stage from a small aero engine (Ref. 7). These experiments were conducted for the purposes of evaluating the second generation sensor's capability of making both clearance and timing measurements on small aero engine hardware. While these foundational experiments were encouraging in that the sensor was able to make measurements on actual aero engine hardware over a relatively low clearance range from 0.10 to $1.50 \mathrm{~mm}$ within the desired accuracy of, it was acknowledged that these results were achieved in a controlled laboratory environment and at a relatively low speed of 200 RPM using a calibration rig. As a follow-on to these experiments and as a means of further maturing the use of microwave blade tip clearance sensors for structural health monitoring applications, the second generation $24 \mathrm{GHz}$ sensors were recently used in the NASA Glenn Research Center's High Precision Spin Rig to evaluate their suitability in 
making blade tip clearance measurements on a 32 blade sub-scale simulated turbine engine disks that were operated at speeds up to 12000 RPM.

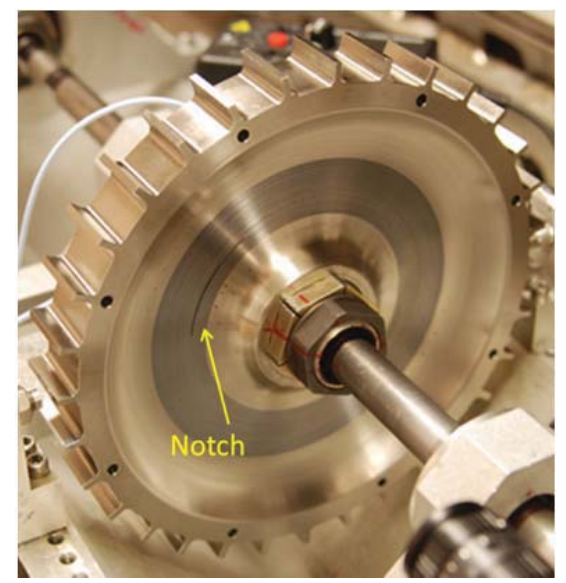

Figure 5. - 32 Blade Nickel Alloy Disk.

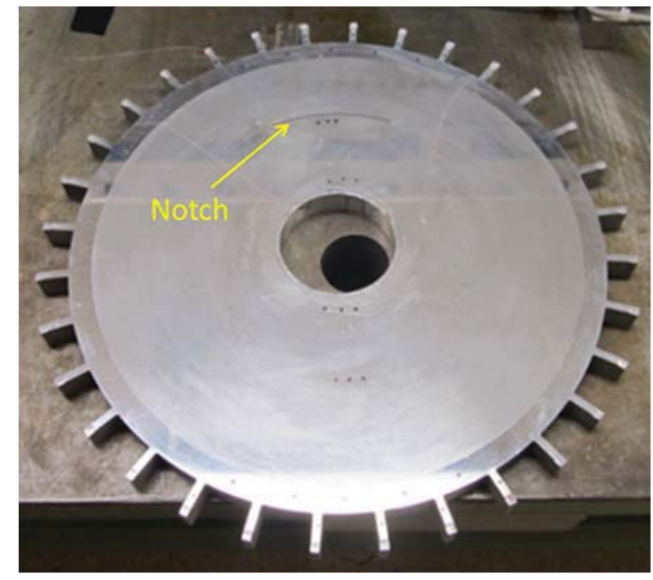

Figure 6. - 32 Blade Aluminum Disk.

As previously indicated, this latest experiment was conducted as a continuation of a series of experiments that have been conducted at the NASA Glenn Research Center over the last several years for the development of new crack detection and propulsion health monitoring techniques (Refs. 8 to 14). The current experiments involved using a combination of $24 \mathrm{GHz}$ microwave and high frequency capacitive blade tip clearance sensors to measure the blade tip clearances and associated vibration responses of sub-scale turbine engine disks that were operated at speeds up to 12000 RPM. Two separate subscale disks were used in the experiment, one made of a Nickel based alloy Hastelloy X (Fig. 5) and the other made of Aluminum (Fig. 6). The two disks were roughly $235 \mathrm{~mm}$ (9.25 in.) in diameter and had been used in previous crackdetection experiments. The Nickel based alloy disk imitated the materials and geometry that are typically used in actual turbine engines, while the Aluminum disk was of a simpler design and better lent itself to foundational or proof-of-concept experiments. Each disk had a $\sim 50 \mathrm{~mm}$ ( $2.0 \mathrm{in}$.) long notch machined into it to simulate a crack. The set-up of the experiment is shown in Figure 7. The purpose of this experiment was two-fold. First, to evaluate the $24 \mathrm{GHz}$ microwave sensors

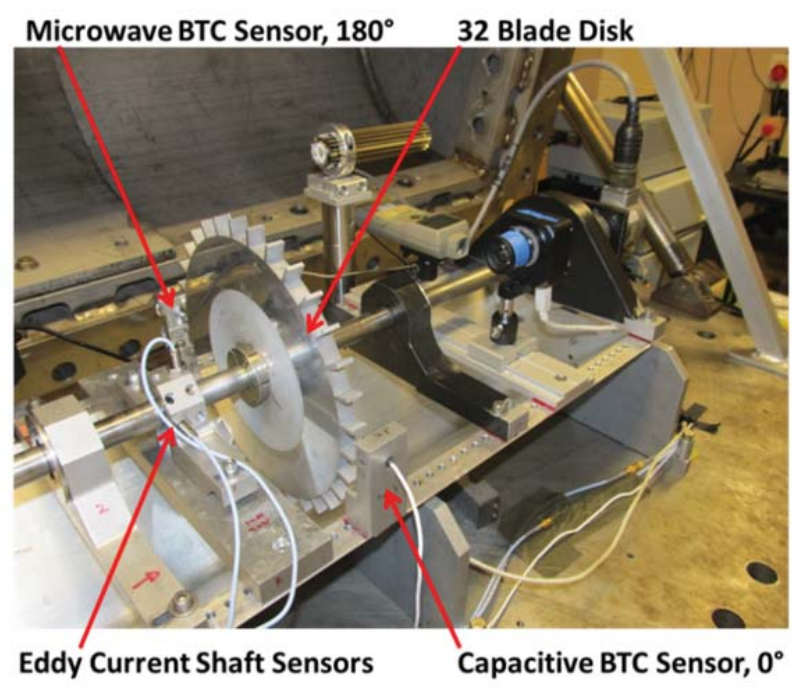

Figure 7. - Experimental Setup, High Precision Spin Rig. 
capability to make accurate and stable blade tip clearance measurements on rotating components operated at speeds up to 12 000 RPM, which is higher than the speeds that were used in prior evaluation tests. Secondly, to determine if the microwave sensors were sensitive and accurate enough to detect the minute changes in the disks whirl amplitude and phase caused by the presence of the simulated crack as predicted by the analytical models. These measurements were compared to those acquired with high frequency capacitive blade tip clearance sensors which have been the standard sensors used in previous crack detection experiments (Ref. 10). A summary of the results from conducting this experiment on the Nickel Alloy Disk is presented in the following sections of this paper. The testing of the Aluminum disk is currently underway and results obtained will be reported on in future publications.

\subsubsection{BLADE TIP CLEARANCE RESULTS FROM NICKEL ALLOY DISK}

A series of 10 test runs were conducted were the disk was operated from 0 RPM to 12000 RPMs with the Nickel Alloy. A typical run profile that was used is shown in Figure 8. Blade tip clearance measurements were made by both a microwave and capacitive blade sensors which were 180 degrees apart. The purpose was to see how the new microwave sensor would track over the entire operating range as compared to the capacitive sensors that had been previously used. The results from one of these experiments using the test profile shown below (Fig. 8) are presented in this paper.

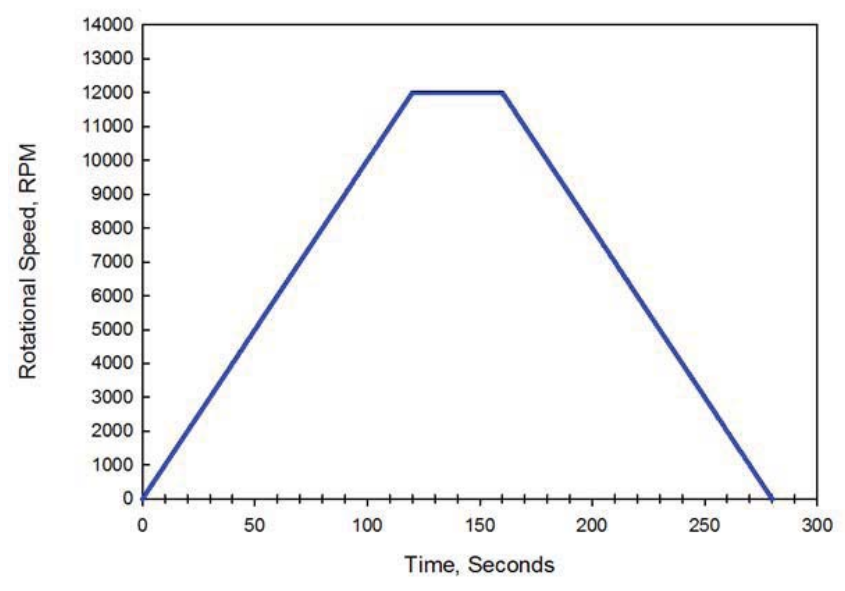

Figure 8. - Typical 12000 RPM Run Profile.

The vibration amplitude, phase Bode plots along with the vibration vector plots of the disk shaft system as measured by the both the capacitive and microwave blade tip clearance sensors from this test run are shown in Figure 9. These plots are used to monitor the structural response of the rotating disk. Changes in vibration as defined by the whirl amplitude and its phase as measured by the blade tip clearance sensors as the disk is transitioned through a range of speeds is used as a means of detecting cracks or flaws in the disk (Refs. 10-14). The data sets were analyzed and processed using an in-house Labviewbased software module. The analysis software was specifically tailored to acquire and post process blade-tip-displacement data. The amplitude shown in the Bode plot displays the first harmonic component of the vibration in inches as a function of disk speed in thousands of revolutions per minute (RPM). The phase of the first harmonic component of the vibration in degrees as a function of disk speed in thousands of revolutions per minute is also shown. The vibration vector plot displays the vibration response in an X-Y (inches vs. inches) format. These plots are the primary analysis method used to determine if the simulated crack could be detected by the vibration response of the system as measured by the respective blade tip clearance sensors. 
From analyzing the plots it is seen that the microwave sensor results closely track the readings from the capacitive sensor. The capacitive sensor measured a vibration amplitude of $\sim 0.15 \mathrm{~mm}(\sim 0.006 \mathrm{in}$.) while microwave sensor measured an amplitude of $\sim 0.10 \mathrm{~mm}(\sim 0.004 \mathrm{in}$.). This slight difference is likely due to the fact that the microwave probes $(\sim 2 \mathrm{~Hz})$ do not sample as fast as the capacitive probes $(\sim 20$ to $200 \mathrm{~Hz})$ and did not capture the precise location of the peak measurement as the disk was quickly transitioned through the first critical speed of 2680 RPM. The phase Bode plots measured by each sensors closely match each other and show the expected 180 degree transition in phase as the disk moves through the first critical speed. While the vibration vector $x-y$ plot defined the by the microwave sensors does not contain as many data points as the one derived by the capacitive sensor, they closely match each other and follow the same overall pattern. From analyzing these plots, with the exception of having less data points, the microwave blade tip clearance sensors performed similar and in-line with the capacitive sensor (Fig. 9).

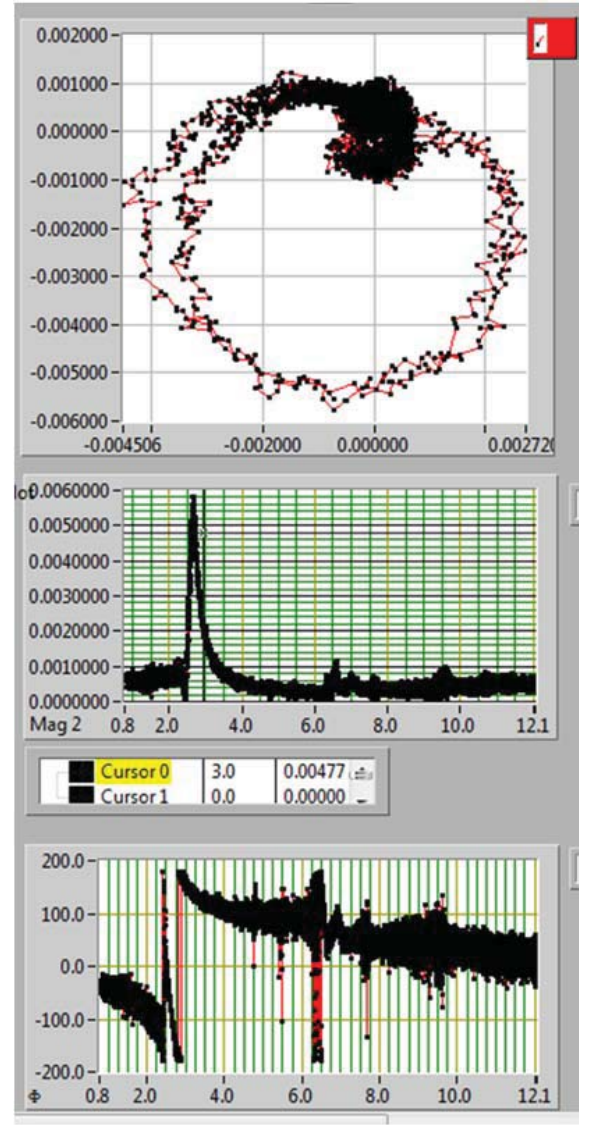

(a)

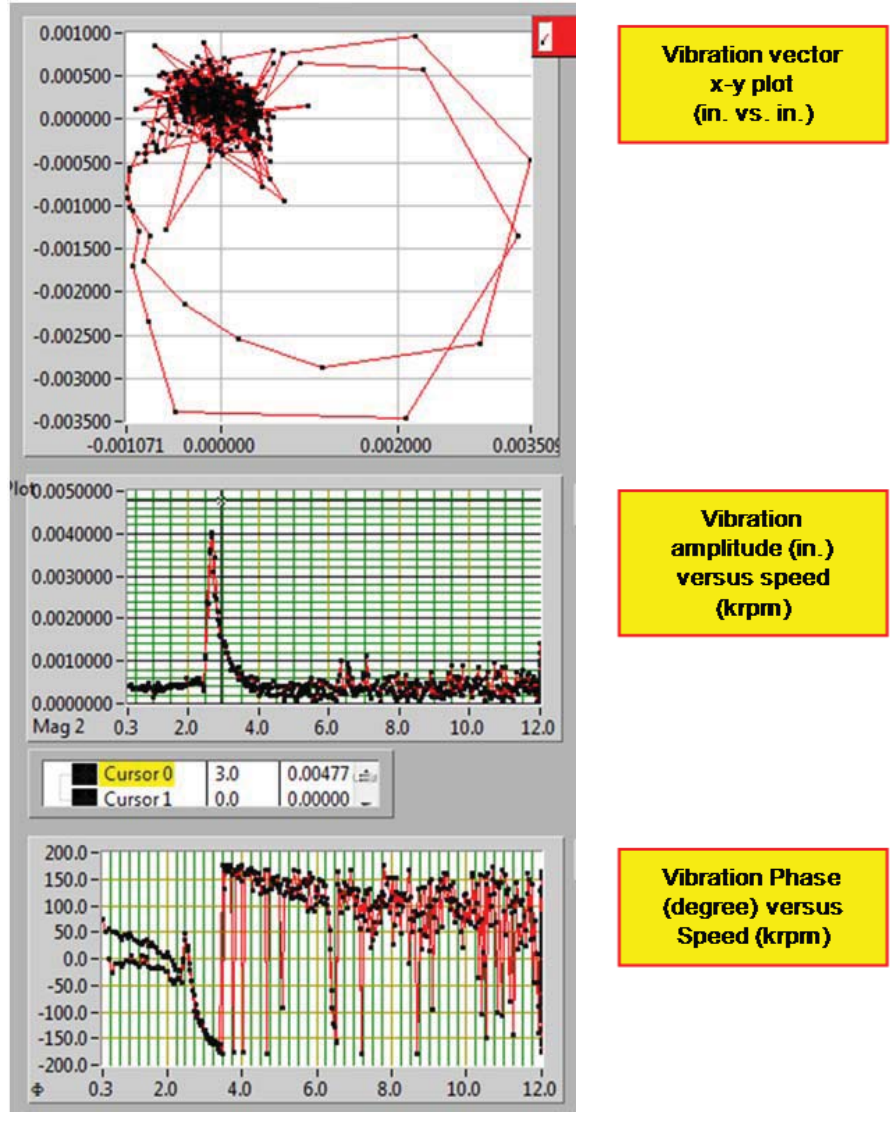

(b)

Figure 9. - 12 000-rpm cycle comparison. (a) Capacitive Sensor Results. (b) Microwave Sensor Results.

A more quantitative analysis was made by examining the blade tip clearance profiles measured by each sensor at several speeds that the disk was tested at. Figures 10-12 show the blade tip clearance profile recorded by each sensor at speeds of 2 680, 4000 and 12000 RPM respectively. Figure 10 shows the profile acquired at 2680 RPM. This speed was chosen as it represents the point where the largest vibration (shaft whirl) amplitude variations are experienced because the disk rotor 
system is transitioning through it first critical speed. From analyzing the blade profile it was seen that each sensor tracked the relative clearances of each blade within reasonable agreement of each other. For the capacitive sensor an average clearance of $\sim 1.32 \mathrm{~mm}(0.052 \mathrm{in}$.) was measured with the difference between the minimum and maximum individual blade tip clearance being $\sim 0.28 \mathrm{~mm}(0.011 \mathrm{in}$.). For the microwave sensor an average clearance of $\sim 1.43 \mathrm{~mm}(0.056$ in.) was measured with the difference between the minimum and maximum measured individual blade tip clearance being $\sim 0.30 \mathrm{~mm}$ (0.012 in.). An offset in the absolute clearance distance of approximately $0.11 \mathrm{~mm}(\sim 0.004 \mathrm{in}$.) on average was observed between the two sensors this is expected and within reason for this experiment. While all attempts were made to install the sensors at a nominal gap of $1.27 \mathrm{~mm}$ to $1.40 \mathrm{~mm}$ ( 0.050 to $0.055 \mathrm{in}$.), differences in the setup of the sensors and blade to blade variations make this difficult to accomplish. The approach for this experiment was to initially gap the sensors as best as could be done at a similar distance and focus on how the sensors varied from these initial positions as they were operated over the entire speed range.

Blade Tip Clearance Profile at 2680 RPM - Nickel Alloy Disk
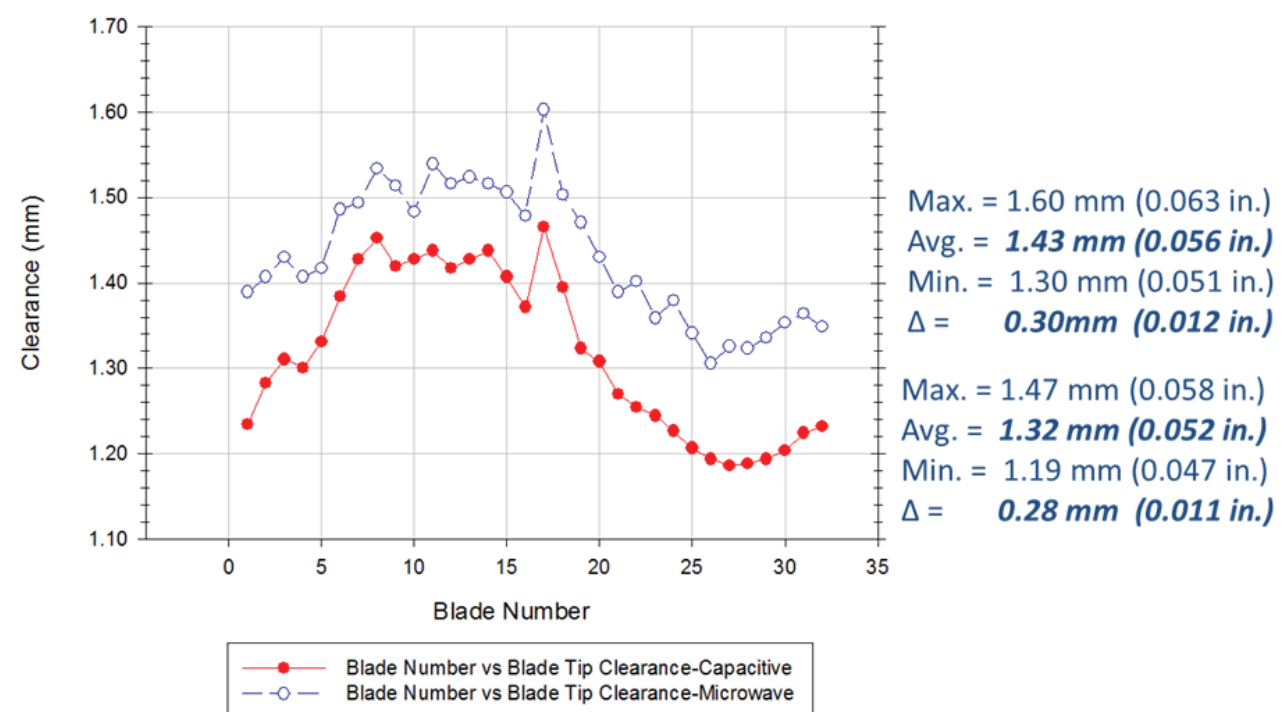

Figure 10. - Blade Tip Clearance Profile at 2680 RPM

Figures 11 and 12 depict the blade tip clearance profiles measured by each sensor at 4000 and 12000 RPM respectively. The speed of 4000 was chosen because it represented a good baseline speed for acquiring reference readings, as it above the critical speed of 2680 RPM, but not yet at the point where the response is influenced by loading and any flaws in the disk. The speed of 12000 RPM was chosen because it was the maximum speed that the disk was operated at and represented the maximum loading condition. From analyzing both of these figures, the profiles for each sensor matched each other and it was shown that the each sensor tracked the relative individual blade tip clearances with-in fairly good agreement of each other. For the capacitive sensor at $4000 \mathrm{RPM}$ an average clearance of $\sim 1.30 \mathrm{~mm}(0.051 \mathrm{in}$.) was measured with the difference between the minimum and maximum individual blade tip clearance being $\sim 0.12 \mathrm{~mm}(0.005 \mathrm{in}$.). For the microwave sensor an average clearance of $\sim 1.39 \mathrm{~mm}(0.055$ in.) was measured with the difference between the minimum and maximum measured individual blade tip clearance being $\sim 0.14 \mathrm{~mm}(0.006 \mathrm{in}$.). Again, the difference in average clearance measurement was due to the difference in the initial gapping of the sensors. The differences between the "delta" (max-min) measurements are nearly within the $+/-0.025 \mathrm{~mm}(+/-0.001 \mathrm{in}$.) accuracy expected for these sensors. 
Blade Tip Clearance Profile at 4000 RPM - Nickel Alloy Disk

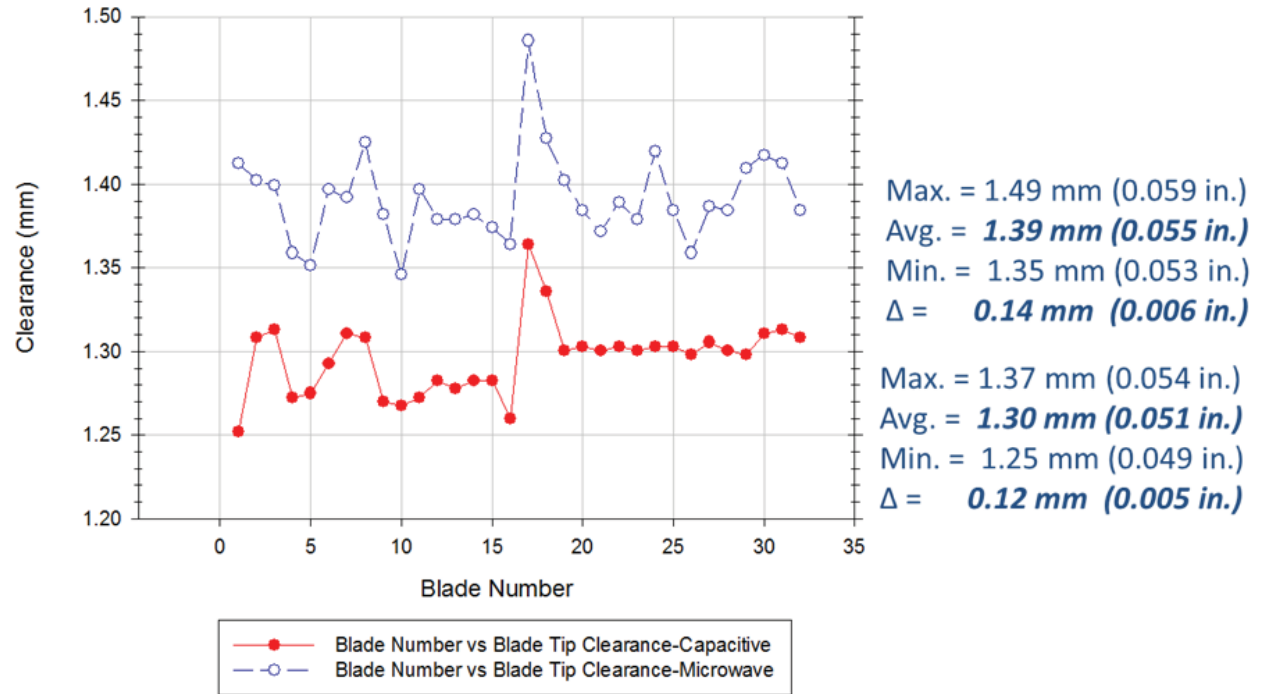

Figure 11. - Blade Tip Clearance Profile at 4000 RPM

Blade Tip Clearance Profile at 12000 RPM - Nickel Alloy Disk

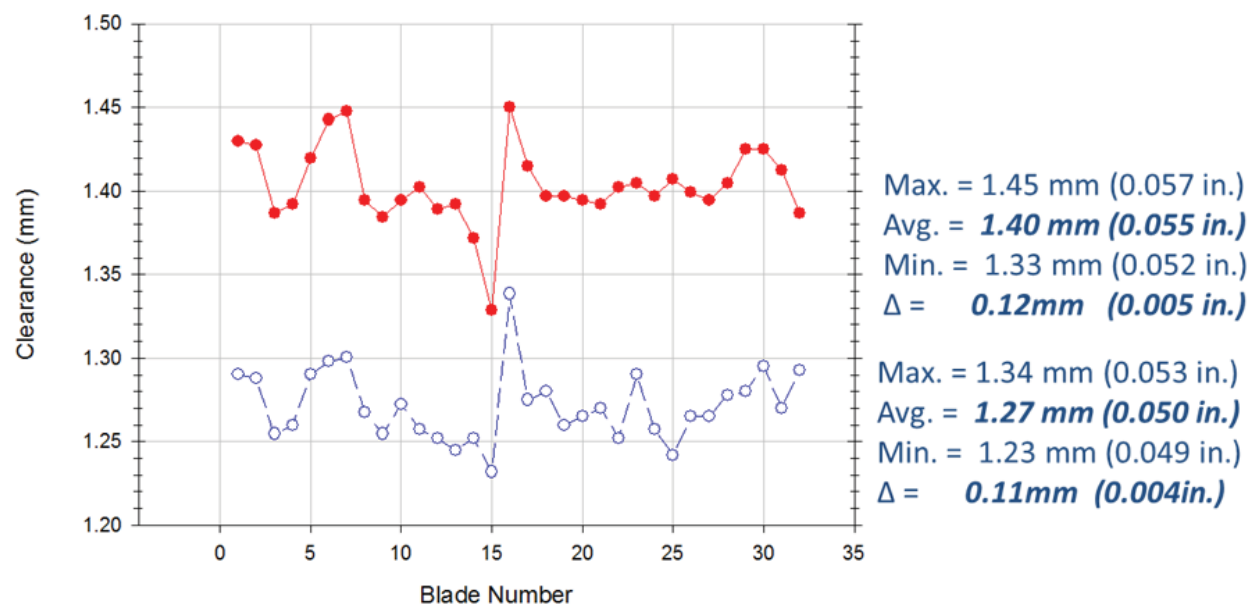

$\longrightarrow-$ Blade Number vs Blade Tip Clearance-Capacitive
$\ldots-\quad$ Blade Number vs Blade Tip Clearance-Microwave

Figure 12. - Blade Tip Clearance Profile at 12000 RPM 
Again, good agreement was observed in the measurements of the individual blade tip clearances in the profile acquired at 12000 RPM (Fig. 12). In this case the capacitive sensor measured an average clearance of $\sim 1.40 \mathrm{~mm}(0.055 \mathrm{in}$.) with a $\sim 0.12 \mathrm{~mm}$ (0.005 in.) difference measured between the minimum and maximum individual blade clearances. The microwave sensor measured an average clearance of $\sim 1.27 \mathrm{~mm}(0.050 \mathrm{in}$.) with the difference measured between the minimum and maximum individual blade clearances being $\sim 0.11 \mathrm{~mm}(0.004 \mathrm{in}$.). This again, is within the expected accuracy of the sensors. It should be noted for this speed, the relative position of the absolute clearance measured by each sensor changed from the baseline condition measurements at 4000 RPM. At 12000 RPM the clearances measured by the capacitive sensor were $\sim 0.10 \mathrm{~mm}(0.004 \mathrm{in}$.) higher than what it measured at 4000 RPM. Similarly, the average clearance measured by the microwave sensor was $\sim 0.12 \mathrm{~mm}(0.005 \mathrm{in}$.) lower than what it measured at $4000 \mathrm{RPM}$. This phenomenon is real and the explanation for this is as follows. It was observed during all of the test runs, at the very high speed conditions of 10000 to 12000 RPM, the overall disk-rotor system pulls or moves in the direction that it is being rotated at. For our test case this was causing the entire disk and shaft system to pull towards the microwave sensor located at the 180 degree position (the back position) and away from the sensor located at the 0 degree position (the front position). Hence, at the high speed conditions, it was observed that the absolute clearances between the microwave sensor and the rotating disk shrank while the clearances between the capacitive sensor in the 0 degree position and the disk grew. Conversely, the opposite of this was observed when the disk was rotated in the opposite direction. Again, this was the first time we had two active sensors working on the rig and it was good to find that this phenomenon occurs so we can take it into account for future experimentation. In summary, based on the analysis presented above, the microwave blade tip clearance sensor proved to provide accurate and reliable readings and matched very well to the capacitive sensors that had been used in previous crack detection experiments.

Once it was found that the microwave sensors were accurate and provided stable readings over the entire disk operational range, the capacitive sensor in the 0 degree position was removed and replaced with another microwave blade tip clearance sensor. Thus, two sensors were installed, one in the original 180 degree position and one in the 0 degree position. A series of four experiments were then conducted operating the disk at speeds from 10000 to 12000 RPM for the purposes of determining if the microwave sensor could detect the presence of the simulated crack, that was previously machined into the disk, using the vibration based monitoring technique described in References 10-14. In this technique the presence of a crack creates a change in the disk-rotor systems center of mass as the disk is operated at high speed conditions. This change translates to an increase in the measured vibration amplitude as a function of rotational speed squared, $\omega^{2}$ (Refs. 13-14).

The vibration amplitude plots derived from the microwave blade tip clearance sensor measurements in both the 0 degree and 180 degree positions are shown in Figure 13. As observed in the plots the amplitude appears to be growing as the speed of operation is increased, which may be indicative of a crack. Curve fitting was attempted in the region above 5000 RPM to look for the expected increase as a function of speed squared, $\omega 2$. This curve fitting was not conclusive and the level of observed growth was not sufficient enough to convincingly state that the sensor detected the presence of the notch, (simulated crack) in the disk. The changes observed were in the $\mu \mathrm{m}$ (sub thousandths of an in.) range and just above the noise floor level. However, this was not viewed as a failure and the results shown in Figure 13 do look promising. Similar results have been obtained using the capacitive sensor. Previous experimentation has found that the Nickel Alloy disk, even with the notch machined in it to simulate a crack, retained its structural integrity with minimal changes experienced even at the high speed, and highly loaded conditions. Hence at the time of the writing of this report, the lighter Aluminum disk is being installed on the spin rig for the purposes of conducting proof-of-concept crack detection experiments using the microwave blade tip clearance sensors. Further analysis is planned for the data acquired from the Nickel alloy disk. The results of this experimentation using the microwave sensors will be presented in the future. 


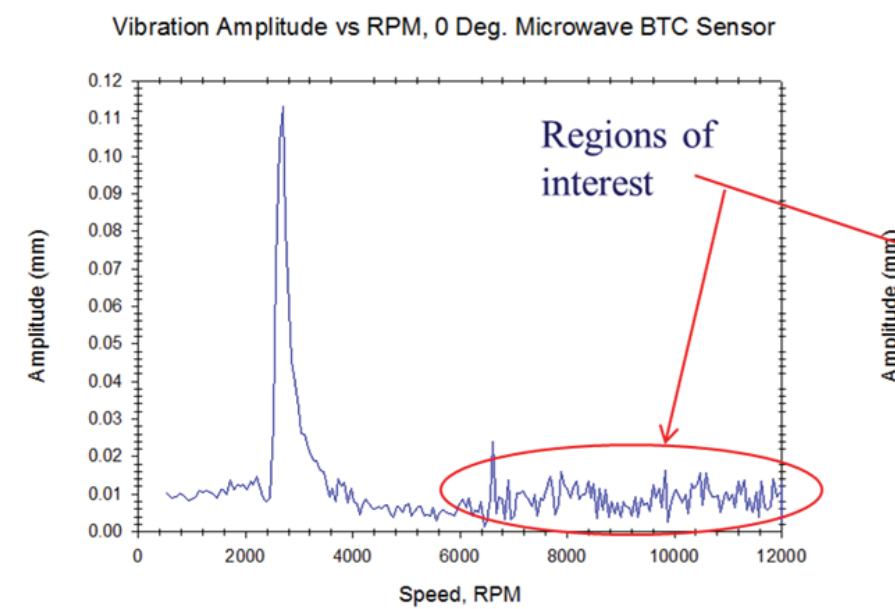

(a)
Vibration Amplitude vs RPM, 180 Deg. Microwave BTC Sensor

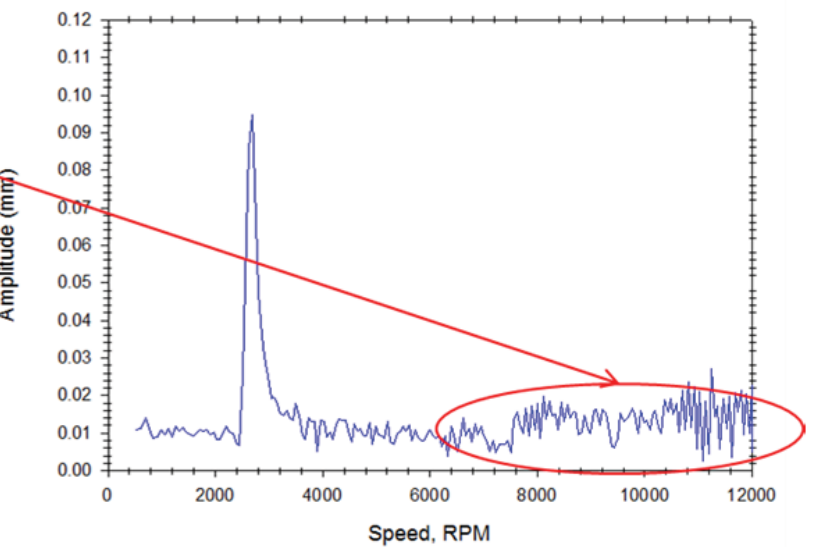

(b)

Figure 13. - 12 000-rpm cycle comparison. Microwave BTC Sensors (a) $0^{\circ}$, Front Location (b) $180^{\circ}$ Back Location

\section{MICROWAVE BASED BLADE VIBRATION SENSOR}

\subsection{SENSOR BACKGROUND AND THEORY}

The novel blade vibration measurement system was delivered to NASA in late 2013 by Mechanical Solutions, Inc (MSI) as part of NASA Aeronautical Research Mission Directorate's (ARMD) 2009 Small Business Innovation Research Program Phase II contract. At a very top level description, the system is a low power microwave sensor based radar system. As in conventional radar, the system transmits a signal and collects the return from a target to measure its motion. However, in the case of this system, the target is a rotating fan, compressor, turbine blade, or any other type of component that is undergoing motion or vibration. Using proprietary algorithms, the system processes the continuous radar data and measures the motion (both rotational and vibrational) of the blade and/or rotating component. This system is innovative in two aspects. First, it can make these measurements at relatively large stand-off distances. Thus eliminating the use of physical sensors, such as strain gages and accelerometer, which are often difficult to implement on rotating components. Secondly, it provides a means of directly measuring the frequency of blade vibration. This is essential in the area of Non-Contact Stress Measurement Sensing (NSMS). Currently, when employing NSMS techniques, the amplitude of blade deflection due to vibration is measured by blade tip timing sensors and the vibrational mode is inferred by analytical model. This parameter (vibrational frequency) can now be directly measured using this new system, and in turn can be used in tandem with blade tip timing sensors to provide a more robust and accurate non-contact stress measurement of a rotating blade. The development of this advancement is outside the scope of this experiment and paper. However, it is planned to investigate this is future developmental efforts.

The goal of this experiment was to simply employ the system on the spin rig and validate its ability to measure the motion, i.e. blade passing frequencies, of the rotating 32 blade disk along with any other vibrational motion that may have been present during the operation of the spin rig. The microwave vibration sensor was aimed at the disk from a distance of $\sim 406 \mathrm{~mm}$ (16 in.) to measure the disks rotational and vibrational motions. The setup of the sensor on the spin rig is shown in Figure 14. The results of this initial validation experiment are presented in the following section. 


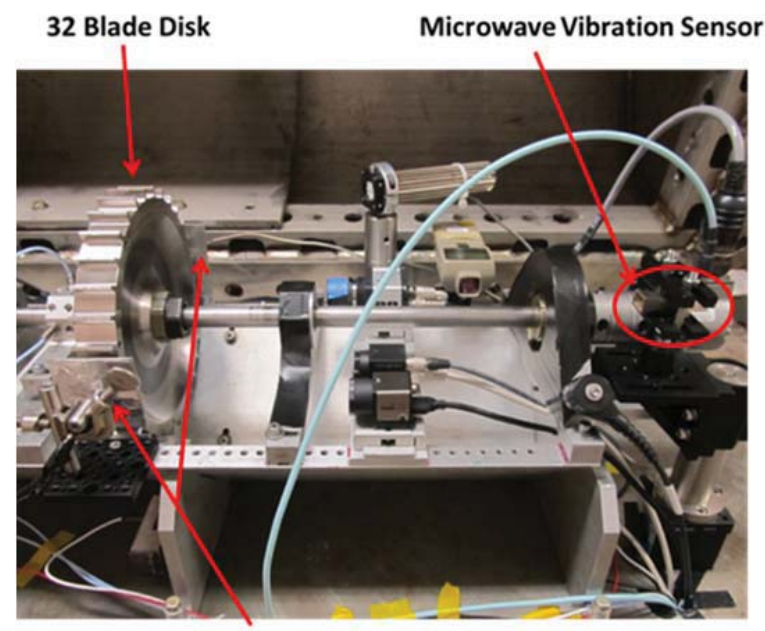

Microwave BTC Sensors

on each side of disk

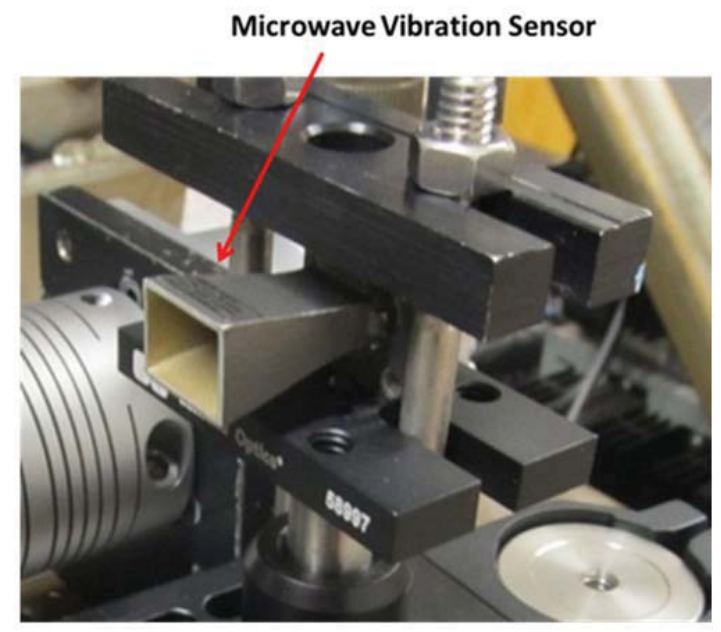

(b)

Figure 14. - Setup of Microwave Vibration Sensor (a) Installation on Test Rig (b) Vibration Sensor

\subsection{EXPERIMENTAL RESULTS}

\subsubsection{ROTATING DISK MEASUREMENTS}

The plot of Figure 15 shows the results acquired from one of the test runs using the non-contact blade vibration measurement sensor. In this case the test run was a simple speed cycle, very similar to the cycle described earlier in the paper. The disk was ramped from 0 RPM to 10000 RPM at an acceleration rate of 100 RPM per second, held at a constant speed of 10000 RPM for 60 seconds, then ramped back down to 0 RPM at a rate of 100 RPM per second. All during this test run a continuous stream of radar data was acquired from the microwave based vibration sensor while it was aimed at the disk from a distance of $\sim 406 \mathrm{~mm}$ (16 in.). The plot on the left is a waterfall plot showing the magnitude and frequency content of the signal acquired from the microwave vibration sensor. The plot shows that the measured vibration frequencies closely track the speed of the disk. The predominate signal frequencies that were measured were the $1 /$ rev frequency (167 $\mathrm{Hz}$ ), the blade passing frequency $(5333 \mathrm{~Hz}$ ) and its second and third harmonics $(10666 \mathrm{~Hz}$ and $16000 \mathrm{~Hz})$. There were also significant signals at $1000 \mathrm{~Hz}$ and $2000 \mathrm{~Hz}$ which were believed to be attributed to vibrations induced by the motor that is used to spin the disk on the rig. Several other test runs were conducted at various speeds and showed similar results where the measured vibration frequencies tracked the speed of the disk and its blade passing frequencies. While this was a simple test of the system, future experimentation is being planned to further use this system to measure blade vibrations on a spinning disk for structural health monitoring purposes. 


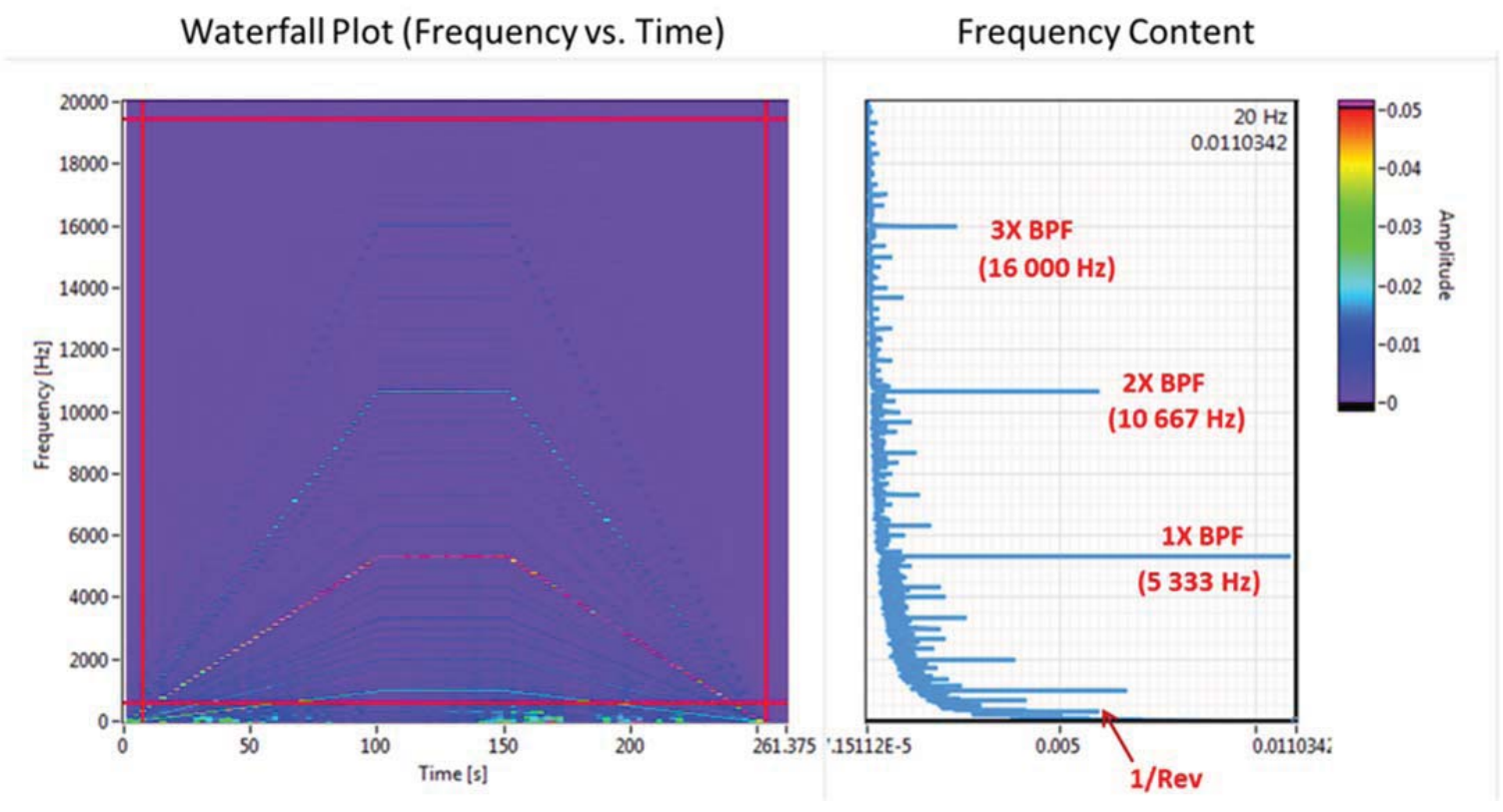

Figure 15. - Waterfall plot, frequency vs. time, 10000 RPM Cycle

\section{CONCLUSION}

The work presented in this a paper represents a summary overview of the on-going research activities that are being conducted at the NASA Glenn Research Center for the development of new sensors and techniques for the structural health monitoring of rotating turbine engine components. The main focus of this effort was to validate the use of a second generation $24 \mathrm{GHz}$ microwave blade tip clearance sensor and a novel microwave blade vibration for making blade tip clearance and structural health measurements in turbine engine applications.

The microwave blade tip clearance sensor technology was found to be a promising option for use in structural health monitoring applications for gas turbine engines. The sensors have been successfully demonstrated on aero engine like hardware. The first generation $5.8 \mathrm{GHz}$ sensors were used on a large Axial Vane Fan and on a NASA Turbofan for the purposes of evaluating the sensor's ability to acquire blade tip clearance data on an aero engine size test article and blades. In the most recent experiment, the second generation $24 \mathrm{GHz}$ sensors were compared to capacitive blade tip clearance sensors in order to assess the microwave sensor's capability to make accurate blade tip clearance measurements on rotating components operated at speeds up to $12000 \mathrm{RPM}$. The results indicated that the microwave sensors were able to provide accurate and reliable readings. Their readings matched very well to capacitive sensors that had been used in previous crack detection experiments. Preliminary work was also accomplished with using these sensors in a vibration based crack detection scheme. While the results were promising, they were not yet conclusive and further work using these sensors for crack detection will be conducted. In addition to what was reported in this paper, a ground test with these sensors installed on a full scale engine was successfully accomplished in the 2013 timeframe. The purpose of this test was to verify that the 
sensors could withstand the harsh environment of a turbine engine. Preliminary data acquired from this engine test are in the process of being analyzed and will be reported on in future conferences/publications.

A microwave blade vibration system was also used to make non-contact frequency of vibration measurements on rotating components. While just a first step and simple test for the system, the microwave vibration sensor was able to successfully measure the blade passing frequency of the rotating disk along with other vibration frequencies associated with the operation of the spin rig. The use of this system will be further refined with the goal of maturing it usage to provide a direct measurement of frequency of blade vibration for non-contact stress measurement (NSMS) applications. More on the usage of this system will be reported on as its capabilities are matured.

\section{REFERENCES}

[1] Kurtoglu, T., Leone, K., Revely, M., and Sandifer, C., "A Study on Current and Emerging Technologies and Future Research Requirements for Integrated Vehicle Health Management," Internal NASA Report, September 2008

[2] Lattime, S.B., and Steinetz, B.M., "Turbine Engine Clearance Control Systems: Current Practices and Future Directions," NASA TM 2002-211794, AIAA-2002-3790, 2002.

[3] Holst, T.A., Kurfess, T.R., Billington, S.A., Geisheimer, J.L., and Littles, J.L, "Development of an OpticalElectromagnetic Model of a Microwave Blade Tip Sensor," AIAA-2005-4377, 2005.

[4] Geisheimer, J.L., Billington, S.A, and Burgess, D.W., "A Microwave Blade Tip Clearance Sensor for Active Clearance Control Applications," AIAA-2004-3720, 2004.

[5] Holst, T.A., "Analysis of Spatial Filtering in Phase-Based Microwave Measurements of Turbine Blade Tips," Master's Thesis, Georgia Institute of Technology, Atlanta, Georgia, August 2005.

[6] Woike, M.R., Roeder, J.W., Hughes, C.E., and Bencic, T.J., "Testing of a Microwave Blade Tip Clearance Sensor at the NASA Glenn Research Center," NASA TM 2009-215589, AIAA-2009-1452.

[7] Woike, M.R., Abdul-Aziz, A., and Bencic, T.J., "A Microwave Blade Tip Clearance Sensor for Propulsion Health Monitoring," NASA TM 2010-216736, AIAA-2010-3308

[8] Woike, M.R., Abdul-Aziz, A., Clem, M.., and Fralick, G., "Optical Strain and Crack-Detection Measurements on a Rotating Disk," NASA TM 2013-216523, SPIE Proceedings Vol. 8693Smart Sensor Phenomena, Technology, Networks, and Systems Integration 2013, 86930M.

[9] Woike, M.R., Abdul-Aziz, A., Fralick, G., and Wrbanek, J.D., "Investigation of a Moiré Based Crack Detection Technique for Propulsion Health Monitoring," NASA TM 2012-217622, Proc. SPIE 8346, Smart Sensor Phenomena, Technology, Networks, and Systems Integration 2012, 834615.

[10] Woike, M.R., Abdul-Aziz, A., "Crack-Detection Experiments on Simulated Turbine Engine Disks in NASA Glenn Research Center's Rotordynamics Laboratory,” NASA TM 2010-216239, AIAA-2010-587

[11] Abdul-Aziz, A., Woike, M.R., Oza, N., and Matthews, B., "Propulsion Health Monitoring of a Turbine Engine Disk Using Spin Test Data," NASA TM 2010-216743, SPIE Proceedings Vol. 7650, Health Monitoring of Structural and Biological Systems 2010, 76501B.

[12] Abdul-Aziz, A., Woike, M. R., Lekki, J. D., and Baaklini, G.Y., "Health Monitoring of a Rotating Disk Using a Combined Analytical-Experimental Approach," NASA/TM-2009-215675, 2009.

[13] Gyekenyesi, A. L., Sawicki, J. T., Martin, R. E., Haase, W. C., and Baaklini, G. Y., "Vibration Based Crack Detection in a Rotating Disk, Part 2-Experimental Results,"NASA/TM-2005-212624/PART2, 2005.

[14] Gyekenyesi, A. L., Sawicki, J. T., and Baaklini, G. Y., "Vibration Based Crack Detection in a Rotating Disk, Part 1—an Analytical Study,” NASA/TM-2003-212624, 2003. 